\title{
The Effectiveness of Public Hospital Governance Model Evaluation and Analysis
}

\author{
Jianhui Yang ${ }^{1}$, Jie Zhao², Yunkai Zhai ${ }^{2}$ \\ Institute of Management Engineering, Zhengzhou University, \\ Zhengzhou, Henan Province, China, 450001
}

Keywords: Public Hospital; Governance; Effectiveness Evaluation

\begin{abstract}
According to the Balanced Scorecard theory and stakeholder theory, this paper points out that the evaluation of the effectiveness of public hospital management mode should be based on in-house effectiveness and the effectiveness of the operation. Article conducts a further demonstration of the effectiveness of public hospital governance, evaluates and analyzes the effectiveness of public hospital governance model.
\end{abstract}

\section{Introduction}

With the further reform of the health system of our country, public hospitals in many regions of our country to implement a variety of modes of governance, many internal and external scholars have made a lot of researches. But there is little study on the evaluation of the effectiveness of the governance model of public hospitals. There is not an ideal model can be adapted to the characteristics of all public hospitals since some factors' influence, such as economic and social development of our country is extremely unbalanced, health care reform and the development process is not the same. So based on public hospitals factors, it is very practical significance to guide the structural reform of our public hospitals, though corresponding evaluation index system and establishing the mathematical model to evaluate the effectiveness of its governance model.

\section{The domestic and foreign public hospital governance models}

Governance model for public hospitals abroad: Japanese and German hospitals are mainly internal control mechanism mode, through the system of internal constraints and motivate the agent's behavior; Australia's public hospitals management is chairman of the board appointed by the state health minister; American Hospital external monitoring mechanism model of governance norms agent behavior by the Hospital external social and market competition to oversee.

From the 80s of the last century, some of our large public hospitals tent to test the joint-stock, ESOP, commission management and other organizational change. In 1997, the State Council promulgated the "Decision on Health Reform and Development", a clear division of medical institutions, leadership structure, operation and management autonomy system; 2001, the State Council held a working meeting of the "three reforms": medical institutions should be an independent legal entity, and the establishment of the specification property rights relations and improve the corporate governance structure; Since 2003, the domestic research focused corporate governance background in public hospitals, the principal-agent problem, the corporate governance structure, governance mechanisms of internal and external constraints, incentives, governance model comparison and around the hospital governance practice summary and other aspects. ${ }^{[1]}$

There are many scholars of the governance structure of public hospitals research governance mechanisms improve governance model aspects of the in-depth discussion and research. But its evaluation of the effectiveness of the governance model and the actual effect is very lacking. Therefore, this article would like to study how to conduct an objective and scientific evaluation of the effectiveness of public hospital governance model, combined with China's specific national conditions and the situation of the health system reform, based on past practice of reform achieved.

The connotation and characteristics of the effectiveness of the governance model of public hospitals 
2.1 The connotation of the effectiveness of the governance model of public hospitals

There is a clear definition on effectiveness in the standard IS09000: "effectiveness refers to the degree of completing planning activities and achieving planning results". Therefore, effectiveness is a kind of measure which means the degree for organization implemented the management mode of operation to realize the goals ${ }^{[2]}$.

The effectiveness of the governance mode refers to the organization can promote the development of the organization and achieve the organization goal easily through governance mode of management reform. As core part of the China's social health service system, public hospital not only needs go for profit, but also should bring into correspondence with social policy, such as protecting the national health, maintaining social stability. There are many researches focus on hospital performance evaluation but only a little research discusses the effectiveness of the governance mode and little achievement. Hospital performance evaluation can not easily estimate the effectiveness of governance mode for public hospital. Consequently, it has very important theoretical value and practical significance for establishing an evaluation index system of management model effectiveness and evaluation method suitable for public hospital.

2.2 The basic characteristics of the effectiveness of the governance model of public hospitals

Evaluation of the effectiveness consists of "to finish the planned activity" and "to achieve the planned result" is base on the connotation of the quality management system and characteristics of its effectiveness .Therefore, the validity of the public hospital governance model should also include the following two fundamental characteristics:

(1)The in-house effectiveness of the governance model means governance model should be reasonable, easy to operate and evaluate. This paper establishes appropriate indicators by the theory of stakeholders to evaluate and analyze the effectiveness of public hospital governance model.

(2)The operational effectiveness of governance mode, namely the feasibility and reliability of the operation of the governance model, it is able to self-improvement based on changes in the internal and external environment, to improve its applicability. This paper utilizes Balanced Scorecard theory of indicators to evaluate and analyze the effectiveness of public hospital governance mode.

The basic characteristic of the two effectiveness of the governance model is the in-house effectiveness of governance model which is the precondition of the validity of its run. However, governance model in-house validity must have to be reflected by the effectiveness of its running. Governance model itself the validity and the effectiveness of its run is unified, interdependent. This article through stakeholder and Balanced Scorecard theory argumentation own validity and effectiveness of the operation of the public hospital governance model and evaluate the effectiveness of the governance model of public hospitals reasonable and scientific.

\section{The establishment of the index system for evaluation of the effectiveness of the governance model of public hospitals}

3.1 The theoretical basis of the evaluation index system of governance model for public hospitals

3.1.1 Stakeholder theory-based evaluation dimensions

The so-called stakeholder refers to those who are in the process of enterprise development, the enterprise production and operation activities can have a significant impact of groups or individuals, the groups or individuals can be internal (such as staff), also may be outside the company (such as suppliers, competitors, etc.). Freeman (1984) proposed stakeholder management model (stakeholder management model, SHM), and points out that they should include "rational, procedures, trade" three levels. Von of spring, bear of road (2007) argues that public hospital reform into the governance structure of stakeholder theory is very necessary. ${ }^{[3]}$ This paper holds that evaluation and analysis of public hospital management mode of the effectiveness of their own, they must pay attention to all public hospital and the interests of stakeholders is ensured.

The division of the stakeholders in the public hospitals, today there are still large controversy. But it is generally agreed that the interests of our public hospitals to the audience should include at 
least the customer (patient), competitors, government departments, suppliers, internal staff, third party billing, non-governmental organizations, etc. YanJun Zhong (2009) argues that public hospital is the main stakeholders including the various government functional departments, patients, hospital staff, hospitals management. WuHao (2010) in the public hospital stakeholders are divided into 3, including primary stakeholders including doctors, patients, secondary stakeholders including government administrative departments, YaoXie suppliers, medical insurance agencies, level 3 stakeholders including medical and health industry group, other ownership hospital. ${ }^{[4]}$ According to the public hospital stakeholder orientation, it is thought that the main stakeholders of public hospital management model including patients, competitors, employees, government, etc.

3.1.2 Evaluation dimensions based on the Balanced Scorecard

Balanced Scorecard from financial, customer, internal operations, and learning and growth of four angles will implement the organization's strategic operational metrics and target value of a new performance management system. Balanced Scorecard was designed to create "strategic guidance" performance management system, in order to ensure the effective execution of corporate strategy.

For the evaluation of the effectiveness of public hospital governance mode, performance management and evaluation is one of the very important aspects and factors. AiTian Yin, XinTai Li (2009) think that we should use the balanced scorecard concept and methods that lead public hospital reform. ${ }^{[5]}$ Balanced Scorecard concept-based performance management system can effectively reflect the effectiveness of the implementation of the governance model of public hospitals, so the analysis and evaluation of the effectiveness of public hospital governance mode.

Based on Balanced Scorecard theory, this paper establishes appropriate refinement indicators from four aspects such as the economic situation, internal processes, learning and development and medical effects feedback. It can concretely reflect the efficiency of the operation of the hospital and the hospital management level; demonstrate the effectiveness of its governance model run.

\section{Conclusion}

By studying the existing public hospital governance model, it is not difficult to find that there is no a universal model of governance applies to all hospitals. Therefore, the public hospital would like to develop a truly suited to their mode of governance must be considering its own characteristics. According to the definition of the effectiveness of the quality management system and its basic characteristics, the paper argues that the effectiveness of public hospital governance model should include the effectiveness of its governance model and the effectiveness of its operation. Thereafter this article describes the use of stakeholder theory to demonstrate the effectiveness of its governance model. And reflect the effectiveness of the operations of its governance model based on the Balanced Scorecard theory.

\section{References}

[1] MingHui Lang, LiQiang Deng. Three main models of corporate governance structure reform of public hospitals [J]. Chinese Hospitals,2009,11(5):15-18.

[2] GuangBo Wei.Summary of our public hospitals, corporate governance mode [J] Chinese Hospitals,2010,9:2-4.

[3] Freeman. Redd. Stockholders and Stakeholders: A New Perspective on Corporate Governance [J]. California Management Review,2010,25: 88-106.

[4] Chun Feng. The need for analysis of public hospital governance structure changes introduced stakeholder theory [J]. Chinese Hospital Management,2012,3:11-12.

[5] AiTian Yin, XinTai Li. Balanced Scorecard concept and methodology to guide the reform of public hospitals [J].Chinese Health Quality Management,2011,4:59-61. 